\title{
Adsorption-Diffusion of Iron in Bovine Keratin
}

\author{
Nicole E. Rinaldi ${ }^{1 *}$, Diego P. FernandeZ ${ }^{1}$, Thure \\ CERLING $^{1}$, AND PAM GROVES ${ }^{2}$ \\ ${ }^{1}$ Univ. of Utah, Salt Lake City, UT, 84102, USA \\ (*correspondence: nicole.rinaldi@utah.edu) \\ ${ }^{2}$ Institute of Arctic Biology, Univ. of Alaska Fairbanks, \\ Fairbanks, AK, 99775, USA
}

Keratin is a common geochemical material frequently utilized in forensic, provenance, archaeological, ecotoxicological and paleoclimatic studies. The incorporation of chemical elements from particles or solutes present in the environment can obscure interpretations when endogenous concentrations are needed, for example when examining exposure to or intake of heavy metals. The interior of thick (>5 mm) modern horn preserves the endogenous chemical composition and it is not impacted by external contamination, which is entirely accumulated within 1 $\mathrm{mm}$ of the surface. However, archaeological keratin may present evidence of long-term continuous alteration: Bison Bob, a recently discovered radiocarbon-dead but extremely well-preserved Alaskan steppe bison (Bison priscus) skeleton, illustrates the progressive incorporation of iron during burial. In this study, we find that diffusion of iron in modern cow horn keratin under anoxic conditions generally follows an adsorption-diffusive profile. This is characterized by high adsorption on the surface of the horn and a concentration-gradient profile in the interior, with a diffusivity of $2.4 \pm 0.9 \times 10^{-10} \mathrm{~cm}^{2} \mathrm{~s}^{-1}$. The physical characteristics of modern horn are analogous to that of Bison Bob, indicating that ancient horn would be expected to follow the same pattern of contamination for iron. A region of the interior of Bison Bob's horn also shows iron concentrations similar to modern uncontaminated keratin, defining a pristine region of the horn. It is thus crucial to use this most pristine keratin for elemental and isotopic analysis. 